ISSN 0103-9954

\title{
QUANTIFICAÇÃO DA BIOMASSA EM UMA FLORESTA ESTACIONAL DECIDUAL EM ITAARA, RS, BRASIL ${ }^{1}$
}

\author{
BIOMASS QUANTIFICATION IN A SEASONAL DECIDUOUS FOREST IN ITAARA, RS, BRAZIL \\ Hamilton Luiz Munari Vogel ${ }^{2} \quad$ Mauro Valdir Schumacher $^{3} \quad$ Peter Trüby $^{4}$ \\ RESUMO
}

O presente trabalho foi realizado no município de Itaara, RS, Brasil, em uma Floresta Estacional Decidual secundária com 70 anos aproximadamente. O estudo teve como principal objetivo quantificar a biomassa acima do solo na floresta. Para tanto, foram abatidas vinte árvores distribuídas em cinco classes de diâmetros. As árvores abatidas foram separadas nas frações, madeira do fuste, casca do fuste, galhos e folhas. Em cada fração, foram retiradas amostras para determinação da umidade, determinado o peso seco de cada componente. A estimativa da biomassa foi obtida pelo uso da equação $\log y=b_{0}+b_{1}$. $\log$ DAP. A produção total estimada de biomassa acima do solo foi de $210,0 \mathrm{Mg} \mathrm{ha}^{-1}$. Nessa estimativa, os galhos corresponderam a $48,8 \%$, a madeira do fuste $43,3 \%$, a casca do fuste $5,4 \%$, e as folhas $2,4 \%$.

Palavras-chave: floresta estacional decidual; biomassa; quantificação; sustentabilidade.

\section{ABSTRACT}

This work was carried out in the city of Itaara-RS, Brazil. The present work has as main objective the quantification of the above ground biomass. To do so, it had been cut down 20 trees and they were distributed in 5 different diameter classes. The trees had been separated in the components stem timber, stem bark, branches and leaves. In each fraction, samples for determination of humidity had been removed, of each component determined the dry weight. The estimate of the biomass was done by the use of the equation $\log \mathrm{y}=\mathrm{b}_{0}+\mathrm{b}_{1} . \log$ DAP. The total production of above ground biomass was $210,0 \mathrm{Mg} \mathrm{ha}^{-1}$. In this estimate, the branches had corresponded to $48,8 \%$ of the above ground biomass, followed by the shafts wood with $43,3 \%$, bark with $5,4 \%$ and the leaves with $2,4 \%$.

Keywords: seasonal deciduous forest; quantification; biomass; sustainability.

\section{INTRODUÇÃO}

No sul do Brasil, a partir do século XIX, as florestas foram gradativamente dando lugar às atividades agropecuárias para suprir a demanda de alimentos e produtos florestais decorrentes do aumento populacional, restando atualmente poucas áreas de floresta nativa. Essas áreas estão localizadas em locais declivosos de difícil acesso.

Nessas regiões, encontram-se hoje em especial pequenas propriedades rurais, onde eventualmente ainda é praticada uma agricultura que consiste do corte da floresta nativa e posterior uso do fogo para limpeza da área. Nessa prática, não é utilizado nenhum tipo de adubo, aproveitando-se os nutrientes da biomassa liberados pela queima do material vegetal.

Em um ecossistema florestal, existe uma grande dinâmica na produção de biomassa. À medida que a floresta se vai desenvolvendo, sobretudo em plantações florestais na fase inicial de crescimento, ocorre uma gradual redução da biomassa da copa das árvores, e simultaneamente verifica-se um aumento na proporção dos componentes madeira e casca (os troncos das árvores geralmente representam em média mais de $80 \%$ da biomassa acima do solo em uma floresta madura) (Schumacher \& Hoppe, 1997).

Dessa maneira, a acumulação de biomassa é afetada por fatores ambientais como luz, temperatura, concentração de $\mathrm{CO}_{2}$ do ar, umidade e fertilidade do solo, e doenças, e também por fatores internos típicos de

1. Parte da tese de doutorado do primeiro autor.

2. Engenheiro Florestal, Dr., Professor da Universidade Estadual do Rio Grande do Sul, Av. Gen. Sampaio, 535, CEP 97541-261, Alegrete (RS). hamiltonvogel@yahoo.com.br

3. Engenheiro Florestal, Dr., Professor Adjunto do Departamento de Ciências Florestais, Centro de Ciências Rurais, Universidade Federal de Santa Maria, CEP 97105-900, Santa Maria (RS). Brasil. Bolsista do CNPq. schuma@ccr.ufsm.br

4. Engenheiro Florestal, Dr., Professor da Albert-Ludwigs-Universität Freiburg, Bertoldstr. 17, 79085 FreiburgAlemanha. peter.trueby@bodenkunde.uni-freiburg.de

Recebido para publicação em 15/12/2005 e aceito em 25/08/2006. 
cada planta, como idade, estrutura e disposição das folhas, distribuição e comportamento dos estômatos, teor de clorofila, entre outros (Kramer \& Kozlowski, 1972).

A estimativa da biomassa de uma floresta nativa pode variar muito com o tipo de floresta e local onde se encontra. Golley et al. (1978), em uma Floresta Tropical Úmida no Panamá, encontraram realidades bastante diferentes de acordo com o local. No sítio da estação úmida, onde tinha uma densidade maior de árvores, a biomassa acima do solo foi maior do que no sítio da estação seca.

Em florestas temperadas, como na região de Solling na Alemanha, a estimativa de biomassa acima do solo em uma floresta de Fagus silvatica com 120 anos de idade, foi de $313 \mathrm{Mg} \mathrm{ha}^{-1}$ (Otto, 1994). Na Ásia, a biomassa média das florestas tropicais nas regiões Sul e Sudoeste foram estimadas em aproximadamente $225 \mathrm{Mg} \mathrm{ha}^{-1}$ (Brown et al.,1991). Porém, no Brasil em uma Floresta Estacional Decidual em Santa TerezaRS, Brun (2004) encontrou uma biomassa total de 186,25 $\mathrm{Mg} \mathrm{ha}^{-1}$, composta por $77,6 \%$ de biomassa acima do solo e $22,4 \%$ pelas raízes.

No presente estudo, foram coletados dados para a quantificação da biomassa acima do solo, em uma Floresta Estacional Decidual secundária em Itaara, RS, Brasil, que visa ao estudo do balanço nutricional e fluxo de elementos nesse ecossistema. Esse trabalho faz parte de uma cooperação entre a Universidade Federal de Santa Maria e a Albert-Ludwigs-Universität Freiburg na Alemanha (PROBRAL-Projeto Brasil Alemanha).

Os estudos envolvendo a quantificação da biomassa constituem a base para o conhecimento do estoque e dinâmica dos nutrientes na floresta, e de como manejar esses ecossistemas de forma sustentável.

Nesse contexto, o trabalho teve como principal objetivo quantificar a produção de biomassa acima do solo em uma Floresta Estacional Decidual em Itaara, RS, Brasil.

\section{MATERIAL E MÉTODOS}

\section{Descrição da área de estudo}

\section{Local}

O trabalho foi realizado em uma fazenda da Brigada Militar (CETRAPA - Centro de Estudos e Treinamento, Reprodução Animal e Proteção Ambiental) no município de Itaara, estado do Rio Grande do Sul, Brasil. O município de Itaara está localizado na Serra de São Martinho, na região físiográfica do Planalto Médio. O local da área experimental tem como coordenadas médias UTM $6731500 \mathrm{~N}$ e 233000 E, com altitude média de $400 \mathrm{~m}$.

\section{Clima}

A região em que o município de Itaara está inserido apresenta a variedade climática Cfa (Classificação climática de Köppen), caracterizada pela ocorrência de chuvas durante todos os meses do ano, possuindo a temperatura média do mês mais quente superior a $22^{\circ} \mathrm{C}$ e a do mês mais frio entre 18 e $-3^{\circ} \mathrm{C}$, com precipitações variando entre 1.700 e 1.800 milímetros anuais (Moreno, 1961).

De acordo com a Plataforma de Coleta de Dados do INPE de São Martinho da Serra, RS (estação mais próxima da área experimental), no ano de 2003 , a temperatura média no local foi de $18,1^{\circ} \mathrm{C}$, e, a precipitação anual de $1.684 \mathrm{~mm}$.

\section{Solo}

O solo da região é caracterizado como Neosolo Litólico Eutrófico típico (Streck et al., 2002). O material de origem é a rocha basáltica. O solo do local do estudo é bastante raso, ficando em torno de $35 \mathrm{~cm}$ de profundidade.

\section{Vegetação arbórea}

De acordo com o inventário realizado na floresta do estudo antes da coleta da biomassa (Longhi et al., 2005), foram encontradas com maior freqüência as espécies arbóreas: Allophylus edulis (A. St.-Hil. et al.) Radlk, Caliandra tweediei Benth., Cupania vernalis Cambess., Dalbergia frutescens (Vell.) Britton, Eugenia hyemalis Cambess., Helietta apiculata Benth., Lithraea brasiliensis Marchand, Lithraea molleoides (Vell.) Engl., Luhea divaricata Mart. et Zucc., Matayba eleagnoides Radlk., Nectandra megapotamica 
(Spreng.) Mez, Ocotea puberula (A. Rich.) Ness, Ocotea pulchella Mart., Parapiptadenia rigida (Benth.) Brenan, Patagonula americana L., Quillaja brasiliensis (A. St.-Hill. et Tul.) Mart., Sebastiana brasiliensis Spreng. e Sebastiania commersoniana (Baill.) L. B. Sm. et Downs, entre outras.

Conforme as informações da Brigada Militar, responsável pela floresta do estudo, estima-se que a floresta tenha em torno de 70 anos de idade. No local, há também indícios de que, no passado, a área já foi explorada (cepas queimadas e cepas ramificadas).

\section{Amostragem da biomassa acima do solo}

Inicialmente foi realizado um inventário florestal da área do estudo. Para tal, foram demarcadas 12 parcelas de $10 \times 10 \mathrm{~m}$ cada, medindo-se todos os DAPs (diâmetro à altura do peito) das árvores com CAP (circunferência à altura do peito) $\geq 10,0 \mathrm{~cm}$, e todas as alturas dos indivíduos e a identificação das espécies arbóreas.

De acordo com as informações obtidas do inventário, foram determinadas cinco classes de diâmetro, de maneira a abranger a maior variação possível do povoamento. O intervalo de classe foi obtido pela fórmula $\mathrm{h}=\mathrm{H} / \mathrm{K}$ em que: $\mathrm{H}=$ amplitude total (DAPmáximo-DAPmínimo) e $\mathrm{K}$ = número de classes, com intervalo de classe igual a 10,36.

No total, foram abatidas vinte árvores, distribuídas dentro das classes de diâmetros, listadas abaixo na Tabela 1. Nessa amostragem, não foram consideradas as lianas e as árvores mortas.

As árvores foram cortadas, independente de classe, com o uso de motosserra. Antes da determinação de sua biomassa, cada árvore foi cubada rigorosamente conforme o método de Smalian, obtendo-se os DAPs e as alturas totais e dos troncos. Após a cubagem, as árvores foram separadas nos componentes, madeira do fuste, casca do fuste, galhos e folhas.

Foi estabelecida como madeira do fuste, a madeira partindo da base do tronco até a primeira bifurcação (conhecida como "forquilha"), sendo então o restante do tronco considerado como galhos. Os galhos não foram amostrados por diâmetro, não sendo divididos em galhos grossos e finos.

TABELA 1: Classes de diâmetro das árvores abatidas na Floresta Estacional Decidual em Itaara, RS, Brasil. TABLE 1: The diameter classes of cut trees in Seasonal Deciduous Forest in Itaara, RS, Brazil.

\begin{tabular}{|c|c|c|c|}
\hline Classe $(\mathrm{cm})$ & N. total indivíduos & N. indivíduos & Espécie \\
\hline \multirow{10}{*}{$3,2-13,5$} & \multirow{10}{*}{11} & 1 & Casearia sylvestris $\mathrm{Sw}$. \\
\hline & & 1 & Helietta apiculata Benth. \\
\hline & & 1 & Eugenia rostrifolia D. Legrand \\
\hline & & 2 & Matayba elaeagnoides Radlk. \\
\hline & & 1 & Sebastiania brasiliensis Spreng. \\
\hline & & 1 & Lonchocarpus nitidus (Vogel) Bent. \\
\hline & & 1 & Cupania vernalis Cambess. \\
\hline & & 1 & Ocotea pulchella Mart. \\
\hline & & 1 & Luehea divaricata Mart. et Zucc. \\
\hline & & 1 & Allophylus edulis (A.St.-Hil.et al.) Radlk. \\
\hline \multirow{3}{*}{$13,6-23,9$} & \multirow{3}{*}{4} & 2 & Matayba elaeagnoides Radlk. \\
\hline & & 1 & Parapiptadenia rigida (Benth.) Brenan \\
\hline & & 1 & Myrsine coriacea (Sw.) R. Br. \\
\hline \multirow{2}{*}{$24-33,6$} & \multirow{2}{*}{3} & 2 & Ocotea pulchella Mart. \\
\hline & & 1 & Ilex brevicuspis Reissek \\
\hline $33,7-44,6$ & 1 & 1 & Ocotea puberula (A. Rich.) Nees. \\
\hline $44,7-55$ & 1 & 1 & Prunus sellowii Koehne \\
\hline
\end{tabular}

Na seqüência, cada componente da árvore foi separado e pesado na sua totalidade, utilizando-se uma balança de gancho. Para as amostras de madeira do fuste e casca, foram retirados três discos do tronco, a $0,10 \mathrm{~cm}$ da altura do fuste, na metade da altura do fuste e no final da altura do fuste. Nesses discos, foi separada a casca da madeira, estimando-se assim a quantidade de casca de todo o fuste.

As coletas de biomassa foram obtidas no período de maio a julho de 2005 . 


\section{Estimativa da biomassa acima do solo}

A estimativa da biomassa de cada componente das árvores de cada uma das parcelas do inventário foi obtida pelo uso da equação $\log \mathrm{y}=\mathrm{b}_{0}+\mathrm{b}_{1} . \log \mathrm{DAP}$, (em que: $\log \mathrm{y}=\log$ aritmo do componente; $\mathrm{b}_{0}$ e $\mathrm{b}_{1}$ $=$ coeficientes da equação, $\log \mathrm{DAP}=$ logaritmo do diâmetro da árvore a $1,30 \mathrm{~m}$ de altura).

\section{RESULTADOS E DISCUSSÃO}

\section{Distribuição diamétrica}

A seleção das árvores para a determinação da biomassa foi em razão dos diâmetros obtidos pelo inventário. Considerando-se as 12 parcelas do inventário, foram medidas um total de 506 árvores com CAP (circunferência a altura do peito) $\geq 10 \mathrm{~cm}$. Os diâmetros foram distribuídas em cinco classes, visualizados na Tabela 2. Nessa Tabela, verifica-se o número de árvores por hectare por classe de diâmetro, em que se constata que o maior número de árvores está na menor classe $(3,2-13,5)$ com $83,6 \%$ destas, com apenas $0,4 \%$ dos indivíduos na maior classe $(44,7-55,0)$, o que é típico em florestas inequiâneas (comportamento exponencial inverso).

Após a distribuição diamétrica, foram abatidas e amostradas vinte árvores. Em conseqüência do número reduzido de árvores nas classes de maiores diâmetros, foi abatida somente uma árvore por classe.

TABELA 2: Classes de diâmetro e freqüência de árvores estimadas e observadas por hectare na Floresta Estacional Decidual em Itaara, RS, Brasil.

TABLE 2: The diameter classes and the frequency of trees estimated and observed per hectare in Seasonal Deciduous Forest in Itaara, RS, Brazil.

\begin{tabular}{c|c|c|c|c}
\hline $\begin{array}{c}\text { Intervalo de classe } \\
(\mathrm{cm})\end{array}$ & $\begin{array}{c}\text { Centro de } \\
\text { classe }\end{array}$ & $\begin{array}{c}\text { Freqüência estimada } \\
\text { ha }^{-1}\end{array}$ & $\begin{array}{c}\text { Árvores } \\
\text { amostradas }\end{array}$ & $\%$ \\
\hline $3,2-13,5$ & 8,35 & 3525 & 11 & 83,6 \\
$13,6-23,9$ & 18,75 & 541,7 & 4 & 12,8 \\
$24,0-33,6$ & 28,8 & 100,0 & 3 & 2,4 \\
$33,7-44,6$ & 39,15 & 33,3 & 1 & 0,8 \\
$44,7-55,0$ & 49,85 & 16,7 & 1 & 0,4 \\
\hline
\end{tabular}

Biomassa acima do solo

A biomassa acima do solo apresentada, nesse estudo, refere-se às árvores em pé com seus componentes: madeira do fuste, casca do fuste, galhos e folhas. Não foram considerados nesse estudo as lianas e as árvores mortas. Na Tabela 3, estão os valores médios da biomassa total por árvore abatida por classe de diâmetro. Observa-se que a maior biomassa se encontra nas maiores classes.

Na Figura 1, está representada a percentagem da biomassa da madeira do fuste com casca por classe de diâmetro. Nota-se que a maior biomassa de madeira do fuste com casca está na segunda classe (43,6\%), e a menor biomassa na última classe com 6,3\%. Esses resultados nos fornecem parâmetros para no caso de uma extração de madeira da floresta em razão do diâmetro aproveitável, o que normalmente ocorre no manejo sustentado de uma floresta. Se for aproveitado, a madeira do fuste com diâmetro acima de $25 \mathrm{~cm}$, será retirada somente $29,5 \%$ da madeira do fuste com casca, permanecendo $70,5 \%$ da madeira na floresta.

TABELA 3: Biomassa total por árvore por classe de diâmetro na Floresta Estacional Decidual em Itaara, RS, Brasil.

TABLE 3: Total biomass per tree per diameter class in Seasonal Deciduous Forest in Itaara, RS, Brazil.

\begin{tabular}{r|c|c|c}
\hline Classe $(\mathrm{cm})$ & Biomassa (kg/árvore) & Desvio & CV $(\%)$ \\
\hline $3,2-13,5$ & 11,8 & 10,6 & 89,5 \\
$13,6-23,9$ & 163,8 & 55,7 & 34,0 \\
$24,0-33,6$ & 458,8 & 222,0 & 48,4 \\
$33,7-44,6$ & $1258,4^{1}$ & - & - \\
$44,7-55,0$ & $1347,8^{1}$ & - & - \\
\hline
\end{tabular}

Em que: 1 = dado referente a uma árvore. 


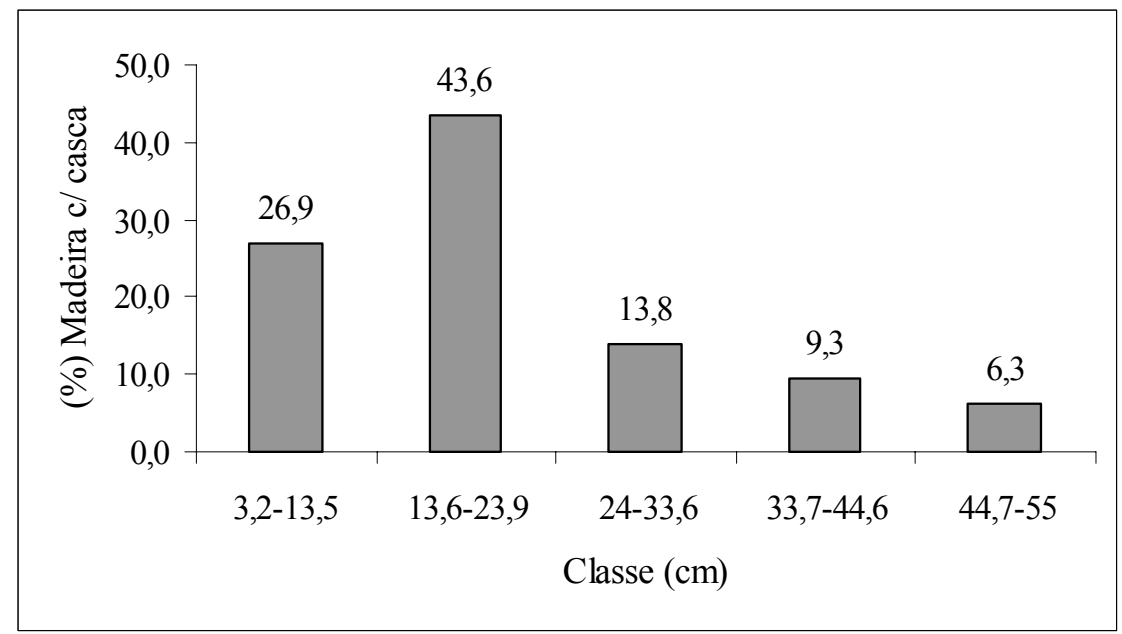

FIGURA 1: Percentagem de madeira do fuste em razão das classes de diâmetro na Floresta Estacional Decidual em Itaara, RS, Brasil.

FIGURE 1: Stem timber percentage in function of diameter class in Seasonal Deciduous Forest in Itaara, RS, Brazil.

De posse dos valores obtidos da determinação da biomassa por classe de diâmetro, com o uso do modelo descrito na metodologia, foi estimada a biomassa seca por hectare dos diferentes componentes das árvores da floresta estudada, visualizados na Figura 2.
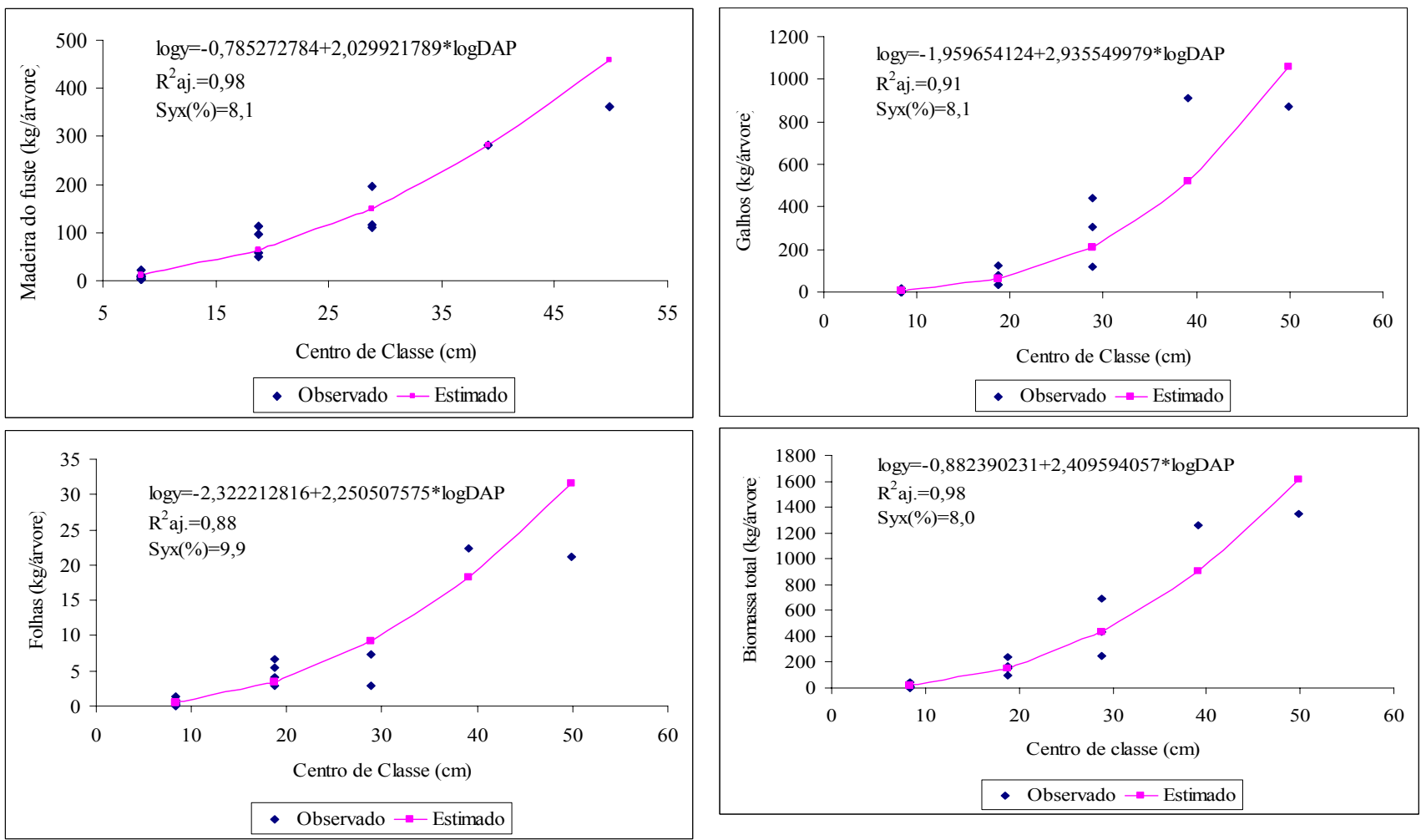

FIGURA 2: Representação gráfica da estimativa dos componentes da biomassa acima do solo na Floresta Estacional Decidual em Itaara, RS, Brasil.

FIGURE 2: Graphical representation of estimate of components of above ground biomass in Seasonal Deciduous Forest in Itaara, RS, Brazil.

Na Figura 2, são apresentados os coeficientes da equação para cada componente e seus respectivos coeficientes de determinação ajustados e o erro-padrão da estimativa em percentagem. Nota-se que foi obtido um ajuste satisfatório na estimativa da biomassa acima do solo para os componentes estudados. Isso pode ser 
constatado pelos valores elevados do coeficiente de determinação ajustados e os baixos erros-padrão percentual da estimativa.

O modelo utilizado nesse trabalho já apresentou boa estimativa da biomassa acima do solo para florestas plantadas de Acácia-negra, Eucalipto e Pinus, (Barichello, 2003; Schumacher et al., 2003). Porém, não se pode afirmar que esse modelo é o mais preciso, pois não foram testados outros modelos em nível de comparação.

$\mathrm{Na}$ Tabela 4, estão apresentadas as estimativas da biomassa acima do solo para cada componente das árvores, por parcela medida no inventário. Nota-se que os valores do coeficiente de variação dos componentes estimados são elevados, sobretudo para o componente "galhos". A alta variação encontrada nos valores estimados deve-se ao fato de que as florestas nativas possuem grande heterogeneidade de espécies, de tamanho, de densidade da madeira, sendo comum encontrar árvores tortas, com fuste pequeno e grande dimensão de copa, o que se reflete em grande variação na determinação da biomassa.

TABELA 4: Biomassa acima do solo estimada por parcela para cada componente das árvores da Floresta Estacional Decidual em Itaara, RS, Brasil.

TABLE 4: Above ground biomass estimated per plot for each component of trees in Seasonal Deciduous Forest in Itaara, RS, Brazil.

\begin{tabular}{c|c|c|c|c|c}
\hline \multirow{2}{*}{ Parcela } & \multicolumn{5}{c}{ Componente $\left(\mathrm{Mg} \mathrm{ha}^{-1}\right)$} \\
\cline { 2 - 6 } & Madeira do fuste & Casca do fuste & Folhas & Galhos & Biomassa Total \\
\hline 1 & 35,3 & 4,6 & 1,8 & 29,2 & 70,9 \\
2 & 126,2 & 15,0 & 7,8 & 206,0 & 354,9 \\
3 & 162,5 & 19,3 & 10,1 & 280,8 & 472,7 \\
4 & 87,8 & 11,3 & 4,8 & 85,7 & 189,6 \\
5 & 115,9 & 14,5 & 6,4 & 125,6 & 262,5 \\
6 & 54,6 & 6,7 & 3,1 & 66,1 & 130,5 \\
7 & 105,9 & 13,5 & 5,6 & 93,2 & 218,2 \\
8 & 79,6 & 10,3 & 4,1 & 62,4 & 156,5 \\
9 & 110,0 & 14,1 & 5,8 & 99,1 & 229,0 \\
10 & 59,8 & 7,9 & 3,0 & 38,6 & 109,3 \\
11 & 89,2 & 11,4 & 4,7 & 79,6 & 184,9 \\
12 & 65,0 & 8,2 & 4,3 & 63,0 & 140,5 \\
\hline Média & 91,0 & 11,4 & 5,1 & 102,4 & 210,0 \\
$\%$ & 43,4 & 5,4 & 2,4 & 48,8 & 100,0 \\
DP & 35,4 & 4,1 & 2,2 & 69,5 & 111,9 \\
CV (\%) & 38,9 & 36,3 & 43,9 & 67,8 & 53,3 \\
\hline
\end{tabular}

Em que: $\mathrm{CV}=$ coeficiente de variação; $\mathrm{DP}=$ desvio padrão.

Observa-se, na Tabela 4, que a média da produção total da biomassa acima do solo estimada foi de 210,0 $\mathrm{Mg} \mathrm{ha}^{-1}$. Nessa estimativa, os galhos corresponderam a 48,8\% da biomassa acima do solo, seguido da madeira do fuste com 43\%, 5,4\% para a casca do fuste, e 2,4\% para as folhas. Assim ficou estabelecida a seguinte ordem de quantidade: galhos $>$ madeira do fuste $>$ casca do fuste $>$ folhas.

Brun (2004) encontrou em uma Floresta Estacional Decidual Secundária com cerca de 50 anos, em Santa Tereza, RS, uma biomassa acima do solo média de 157,6 $\mathrm{Mg} \mathrm{ha}^{-1}$ (incluindo extrato arbustivo, herbáceo e lianas), sendo sua maior parte formada pela madeira $(52,41 \%)$ seguido pelos galhos $(28,4 \%)$. Ainda conforme esse autor, a biomassa das parcelas analisadas variou de $82,7 \mathrm{Mg}$ ha $^{-1}$ a $232,5 \mathrm{Mg} \mathrm{ha}^{-1}$, indicando a alta variabilidade da estimativa nas parcelas, fato esse também observado no presente estudo (Tabela 3).

Já em uma Floresta Ombrófila Mista Montana em General Carneiro no Paraná, Caldeira (2003) estimou uma biomassa acima do solo com DAP $\geq 10 \mathrm{~cm}$, de $210,4 \mathrm{Mg} \mathrm{ha}^{-1}$, assim distribuída: galhos $(45,3 \%)>$ madeira do fuste $(41,1 \%)>$ casca $(10,3 \%)>$ folhas $(2,4 \%)$.

Golley et al. (1978), em uma Floresta Tropical Úmida no Panamá, constataram realidades bastante diferentes de acordo com o local, uma vez que o sítio para a estação úmida tinha também uma densidade 
maior de árvores. Assim a biomassa acima do solo foi de $367,9 \mathrm{Mg} \mathrm{ha}^{-1}$ para o sítio da estação úmida, e 263,5 $\mathrm{Mg} \mathrm{ha}^{-1}$ para o sítio da estação seca.

Uma das dificuldades encontradas nos estudos de biomassa em florestas nativas é estabelecer o ponto do fuste, partindo do qual ele deve ser considerado como galho. Nesse estudo, a biomassa do componente madeira do fuste foi estabelecida até o ponto do tronco em que ocorre a primeira bifurcação do tronco (também conhecida como "forquilha"), sendo então o restante considerado como galhos, não sendo levado em conta o seu diâmetro.

Nas florestas nativas no sul do Brasil, é comum a ocorrência de árvores com pequena altura de fuste, e grande proporção de copa, o que acarreta em valores elevados de biomassa dos galhos, como os observados no presente estudo.

\section{CONCLUSÕES}

$\mathrm{Na}$ floresta estudada, a produção total de biomassa acima do solo foi estimada em $210,0 \mathrm{Mg} \mathrm{ha}^{-1}$.

No estágio atual de crescimento da floresta (em torno de 70 anos), ficou constatado que a maior percentagem de biomassa acima do solo está nos galhos (48,8\%), seguido da madeira do fuste com 43,3\%.

Os componentes com menor participação relativa da biomassa acima do solo foram a casca do fuste com $5,4 \%$, e as folhas com $2,4 \%$.

\section{REFERÊNCIAS BIBLIOGRÁFICAS}

BARICHELLO, L. R. Quantificação da biomassa e dos nutrientes em floresta de Acacia mearnsii De Wild. na região sul do Brasil. 2003. 58f. Dissertação (Mestrado em Silvicultura) - Universidade Federal de Santa Maria, Santa Maria, 2003.

BROWN, S.; GILLESPIE, A. J. R.; LUGO, A. E. Biomass of tropical forests of south and southeast Asia. Can. J. For. Res., v. 21, p.111-117, 1991.

BRUN, E. J. Biomassa e nutrientes na floresta Estacional Decidual, em Santa Tereza, RS. 2004. 136f. Dissertação (Mestrado em Silvicultura) - Universidade Federal de Santa Maria, Santa Maria, 2004.

CALDEIRA, M. V. W. Determinação de biomassa e nutrientes em uma floresta ombrófila mista montana em General Carneiro, Paraná. 2003. 176f. Tese (Doutorado em Engenharia Florestal) - Universidade Federal do Paraná, Curitiba, 2003.

GOLLEY, F. B. et al. Ciclagem de minerais em um ecossistema de floresta tropical úmida. São Paulo: EPU : Ed. da USP, 1978. 256p.

KRAMER, R. J.; KOSLOWSKI, T. T. Fisiologia das árvores. Lisboa: Fundação Kalouste Goulbenkian, 1972. 745p.

LINDMAN, C. A. M.; FERRI, M. G. A vegetação no Rio Grande do Sul. Belo Horizonte, 1974. 390p.

LONGHI, S. J. et al. Caracterização fitossociológica de um fragmento de floresta estacional decidual em Itaara, RS. Santa Maria, 2005. (Relatório Técnico)

MORENO, J. A. Clima do Rio Grande do Sul. Porto Alegre: Secretaria da Agricultura, 1961. 73p.

OTTO, H. J. Waldökologie. Stuttgart : Ulmer, 1994. 391p.

SCHUMACHER, M. V.; HOPPE, J. M. A complexidade dos ecossistemas. Porto Alegre: Pallotti, 1997. 50p.

SCHUMACHER, M. V. et al. Quantificação do carbono e dos nutrientes em florestas de eucalipto de diferentes idades. Santa Maria: UFSM/CCR/DCF/FATEC, 2003. 112p. (Relatório de Pesquisa.)

STRECK, E. V. et al. Solos do Rio Grande do Sul. Porto Alegre, RS: Emater/RS : UFRGS, 2002. 107p. 